\title{
Electromagnetic oscillations of the Earth's upper atmosphere (review)
}

\author{
A. G. Khantadze ${ }^{1}$, G. V. Jandieri ${ }^{2,3}$, A. Ishimaru ${ }^{4}$, T. D. Kaladze ${ }^{5}$, and Zh. M. Diasamidze ${ }^{2}$ \\ ${ }^{1}$ Metrology and Hydrology Department, Tbilisi State University, Chavchavadze Avenue, 1, 0128, Tbilisi, Georgia \\ ${ }^{2}$ Physics Department, Georgian Technical University, Kostava Street, 77, 0175, Tbilisi, Georgia \\ ${ }^{3}$ Institute of Cybernetics, S. Euli street, 5, 0186, Tbilisi, Georgia \\ ${ }^{4}$ Department of Electrical Engineering, University of Washington, FT-10 Seattle, Washington 98195, USA \\ ${ }^{5}$ I. Vekua Institute of Applied Mathematics, Tbilisi State University, 2 University str., 0186 Tbilisi, Georgia
}

Received: 5 March 2010 - Revised: 11 June 2010 - Accepted: 11 June 2010 - Published: 1 July 2010

\begin{abstract}
A complete theory of low-frequency MHD oscillations of the Earth's weakly ionized ionosphere is formulated. Peculiarities of excitation and propagation of electromagnetic acoustic-gravity, MHD and planetary waves are considered in the Earth's ionosphere. The general dispersion equation is derived for the magneto-acoustic, magnetogravity and electromagnetic planetary waves in the ionospheric E- and F-regions. The action of the geomagnetic field on the propagation of acoustic-gravity waves is elucidated. The nature of the existence of the comparatively new largescale electromagnetic planetary branches is emphasized.
\end{abstract}

Keywords. Radio science (Waves in plasma)

\section{Introduction}

In the present review, both new and known branches of electromagnetic oscillations of the Earth's weakly ionized upper atmosphere (ionosphere) are considered; the Earth's magnetic field ionospheric E- and F-layers consisting of electrons, ions and neutral particles. In the upper atmosphere, all wavy varieties can be divided into a relatively small, mesoscale (with wavelengths $<10^{3} \mathrm{~km}$ ) and large, synoptic-scale (with wavelengths in the region of $10^{3}-10^{4} \mathrm{~km}$ ) perturbations. In the first class of waves belong the acoustic, inertio-gravitational and MHD (Alfvén and magnetoacoustic) waves, while the second class of waves contains planetary Rossby waves and magnetogradient waves, which are caused by the latitudinal inhomogeneity of both the angu-

Correspondence to: G. V. Jandieri (jandieri@access.sanet.ge) lar velocity of the Earth's rotation and the geomagnetic field. Similarly, with the troposphere, we will call the large-scale waves weather-forming waves since they carry over largescale vortices of cyclonic and anticyclone nature. We will not consider the high-frequency electromagnetic waves of plasma. During the selection of material, of course, the personal scientific interests of the authors played quite important role.

It is well known that the set of thermohydrodynamic partial differential equations of the lower atmosphere (troposphere) is of the fifth order over the time derivative (the system contains time derivatives from three velocity components, pressure and density). Consequently, the solution of the Cauchy problem for this system of equations requires the setting of the five fields of meteorological elements at the initial time. According to the observations (Kelley, 1989; Alperovich and Fedorov, 2007), wavy motions in the troposphere, propagating under the arbitrary initial conditions, can be clearly divided into relatively slow (synoptic) and fast motions. Slow wavy structures in the troposphere are always of a large-scale (with wavelength $\lambda \sim 10^{3}-10^{4} \mathrm{~km}$ ) and longperiod (from two days to two weeks and more) nature and move in the atmosphere with the velocities exceeding zonal winds $\left(5-20 \mathrm{~m} \mathrm{~s}^{-1}\right)$. These wavy perturbations (planetary Rossby waves) actually determine regional weather in the troposphere. Fast wavy motions, which usually are of a short-period (from several minutes to an hour) and smallscale $\left(\lambda \leq 10^{3} \mathrm{~km}\right)$ nature, propagate in the troposphere with a sound velocity and are generated due to the compressibility and temperature stratification of the atmosphere.

The synoptic processes reduction of the time derivative order of the set of partial differential equations of the troposphere dynamics (from the fifth to the first) is justified

Published by Copernicus Publications on behalf of the European Geosciences Union. 
by the fact that the fast wavy motions in the slow weatherforming processes create only "meteorological noise" and, therefore, they need to be filtered in advance. Indeed, in following numerous observations (Kelley, 1989; Alperovich and Fedorov, 2007), the real atmosphere quickly restores the violation of both quasi-static (during several minutes) and quasi-geostrophic (roughly during one hour) states. Thus, for the synoptic processes (two weeks and more), it may be considered that the atmosphere always is in the quasi-static and quasi-geostrophic states. As it was shown (Monin and Obukhov, 1958; Yaglom, 1953), when observing these conditions four frequencies of acoustic-gravity waves (AGW) fall out from the dispersion equation and only the frequency of planetary Rossby waves remains. Taking into account the fact that the vector of the Earth's angular velocity $\omega_{0}(\chi)(\chi$ is the latitude) is always directed from the south to the north, the latitudinal gradient of the Coriolis force generates largescale wavy perturbations in the atmosphere, which move along the parallels in the one direction (westwards) only.

Unlike the troposphere, the set of MHD equations of the electrically conducting ionosphere is of the eighth order over the time derivative (besides the five meteorological elements, the system now contains also three components of induced magnetic field). Therefore, the wavy processes in the upper atmosphere have both a hydrodynamic and electromagnetic nature. In the large-scale wavy processes, the induced magnetic field enriches temporal spectrum with high frequencies and, therefore, planetary waves in the ionosphere become both slow and long-period (hydrodynamic Rossby-type planetary waves: Tolstoy, 1967; Khantadze, 1967) and fast and short-period as well. The new, fast planetary waves have the electromagnetic nature with relatively high frequencies $\left(10^{-3}-10^{-4} \mathrm{~s}^{-1}\right)$ and move in the ionosphere with a velocity of more than $1 \mathrm{~km} \mathrm{~s}^{-1}$. First, these waves were theoretically discovered by Khantadze $(1986,1999,2001)$. Slow and fast planetary waves are generated in the ionosphere by the latitudinal gradient of the electromagnetic Ampere's force $\boldsymbol{F}_{A}=\boldsymbol{j} \times \boldsymbol{H}_{0} / c \rho$, where $\rho$ is the density of a neutral component, $c$ is the light speed, $\boldsymbol{j}$ is the current density, and $\boldsymbol{H}_{0}\left(r, \chi^{\prime}\right)$ is the geomagnetic field; $r$ is a distance from the Earth's center, $\chi^{\prime}$ is the geomagnetic latitude (in the sequel we assume that the geographic and geomagnetic latitudes are coinciding). The geomagnetic field also generates small and medium-scale waves: magneto-acoustic and Alfvén waves. In the ionosphere, magneto-acoustic waves are generated by the elasticity of the geomagnetic lines of force. These are fast waves (with the propagation velocity more that $1 \mathrm{~km} \mathrm{~s}^{-1}$ ) and short-period (of the order of 5-20 min). Alfvén waves, with phase velocity depending on the orientation of the wave vector $\boldsymbol{k}$ with respect to the geomagnetic field $\boldsymbol{H}_{\mathbf{0}}$, are generated due to the tension of the geomagnetic lines of force and, as it will be shown below, can be very slow $\left(10 \div 50 \mathrm{~m} \mathrm{~s}^{-1}\right)$ and long-period $(1 \div 2$ days), when the wave vector $\boldsymbol{k}$ is almost transversal to $\boldsymbol{H}_{\mathbf{0}}$ and fast, when vectors $\boldsymbol{k}$ and $\boldsymbol{H}_{\mathbf{0}}$ are parallel.
It follows that the traditional method of wave filtration used in the troposphere does not work under the ionospheric conditions. Therefore, hereafter we will consider planetary waves, and small and medium-scale waves independently. Certainly, at the same time, some important effects of interaction of the waves with different scales are lost (Khantadze et al., 1982), however, in linear approximation such consideration seems correct.

The review is organised in the following fashion: in Sect. 2 the basic equations for the electromagnetic ionospheric oscillations are formulated and discussed. The main peculiarities of the propagating waves are considered and the necessity of the consideration of their electromagnetic nature is emphasized. The nature of the slow MHD waves in the ionosphere is explained in Sect. 3. In Sect. 4, the influence of the geomagnetic field on the propagation of AGW in the ionosphere is considered. Comparatively, a new class of ULF electromagnetic planetary waves in the ionospheric E- and F-regions is clarified in Sect. 5. Our discussions and conclusions can be found in Sect. 6 .

\section{Equations of electromagnetic ionospheric oscillations}

Let us consider the electrically conducting, compressible and stratified ionospheric medium where wavy processes occur polytropically with the polytrophic coefficient $æ$. Taking into account Hall's effect, the basic MHD equations of the ionosphere can be presented in the following form (Khantadze, 1971):

$$
\begin{aligned}
& \rho \frac{d \boldsymbol{V}}{d t}=-\nabla P+\rho \boldsymbol{V} \times 2 \boldsymbol{\omega}_{\mathbf{0}}+\rho \boldsymbol{g}+\frac{1}{4 \pi} \nabla \times \boldsymbol{H} \times \boldsymbol{H}, \\
& \frac{\partial \rho}{\partial t}+\nabla \cdot \rho \boldsymbol{V}=0, \\
& \frac{\partial \boldsymbol{H}}{\partial t}=\nabla \times \boldsymbol{V} \times \boldsymbol{H}-\delta \nabla \times\left(\alpha \frac{1}{4 \pi} \nabla \times \boldsymbol{H} \times \boldsymbol{H}\right), \\
& \frac{d P}{d t}=\mathfrak{x} \frac{\mathrm{P}}{\rho} \frac{d \rho}{d t} .
\end{aligned}
$$

Here $\boldsymbol{V}$ and $\boldsymbol{H}$ are vectors of the fluid velocity and magnetic field, respectively. $P$ and $\rho$ are pressure and density of the neutral component of the ionosphere, $g$ is the vector of gravitational acceleration, $\alpha=c / e N$ is the Hall's parameter, $c$ is the speed of light, $e$ is the elementary charge, $N$ is the concentration of the ionospheric plasma. Here we introduce the dimensionless parameter $\delta=1$ in the presence of Hall's effect (E-region of the ionosphere) and $\delta=0$ when Hall's effect disappears (F-region).

Firstly, let us note that the geomagnetic field $\boldsymbol{H}_{\mathbf{0}}$ satisfies Maxwell's equations: $\nabla \times \boldsymbol{H}_{\mathbf{0}}=0, \nabla \cdot \boldsymbol{H}_{\mathbf{0}}=0$ in the ionosphere and, therefore, in the ground state the system (1) passes into the set of equations on basic motion of the ordinary atmosphere. In the ionosphere the geomagnetic field $\boldsymbol{H}_{0}$ generates an electric field (dynamo field), caused by the 
wind mechanism $\boldsymbol{E}_{\mathbf{0}}=\boldsymbol{V}_{\mathbf{0}} \times \boldsymbol{H}_{\mathbf{0}}$, here $\boldsymbol{V}_{\mathbf{0}}$ is the motion velocity in the ground state.

In the present paper, we will not investigate the influence of the medium's ground state motion on the oscillations, but we will consider the equilibrium state when pressure $\bar{P}$ and density $\bar{\rho}$ depend only on the z-coordinate and are connected to each other by the static equilibrium equation $\partial \bar{P} / \partial z=-\bar{\rho} g$.

If we neglect, for simplicity, the action of the Coriolis force and Hall's effect and linearized the set of equations (Eq. 1) with respect to the equilibrium state, we easily get one vector equation for the perturbed velocity $\boldsymbol{V}$ :

$$
\begin{aligned}
\frac{\partial^{2} \boldsymbol{V}}{\partial t^{2}}= & c_{\mathrm{s}}^{2} \nabla(\nabla \cdot \boldsymbol{V})+\nabla(\boldsymbol{V} \times \boldsymbol{g})+(\mathfrak{x}-1) \boldsymbol{g} \nabla \cdot \boldsymbol{V} \\
& -\frac{1}{4 \pi \rho_{0}} \boldsymbol{H}_{\mathbf{0}} \times \nabla \times \nabla \times \boldsymbol{V} \times \boldsymbol{H}_{\mathbf{0}},
\end{aligned}
$$

where $c_{\mathrm{s}}^{2}=\mathfrak{P} \bar{P}_{a} / \bar{\rho}_{a}=\mathfrak{g} H$ is the square of the sound velocity and $H=R \bar{T}_{0} / g$ is the height of the homogeneous atmosphere. Further it is assumed that $\bar{T}_{0}=$ const.

Derivation of the general dispersion equation from Eq. (2) is quite a difficult problem, however if we introduce $\theta_{k}-$ angle between wave vector $\boldsymbol{k}$ and vertical, $\theta_{H}$ - angle between the geomagnetic field $\boldsymbol{H}_{\mathbf{0}}$ and vertical, $\theta_{k H}$ - angle between vectors $\boldsymbol{k}$ and $\boldsymbol{H}_{\mathbf{0}}$, the general dispersion equation can be obtained in the form (McLellan and Winterberg, 1968; Khantadze, 1973):

$$
\begin{aligned}
\left(\omega^{2}-\omega_{a}^{2} \cos ^{2} \theta_{k}\right) & {\left[\omega^{4}-\omega^{2}\left(\omega_{\mathrm{s}}^{2}+\omega_{a}^{2}+i \frac{\omega_{\mathrm{s}}^{2}}{k H} \cos \theta_{k}\right)\right.} \\
& +N^{2} \omega_{\mathrm{s}}^{2} \sin ^{2} \theta_{k}+i \frac{\omega_{\mathrm{s}}^{2} \omega_{a}^{2}}{k H} \cos \theta_{H} \cos \theta_{k H} \\
& \left.+\omega_{\mathrm{s}}^{2} \omega_{a}^{2} \cos ^{2} \theta_{k H}\right]=0 .
\end{aligned}
$$

Here $\omega_{a}^{2}=V_{a}^{2} k^{2}, \omega_{\mathrm{s}}^{2}=c_{\mathrm{s}}^{2} k^{2}, N^{2}=\left(g^{2} / c_{\mathrm{s}}^{2}+g / \bar{\rho} d \bar{\rho} / d z\right)=$ $g(1-1 / \mathfrak{x}) / H$ are squared frequencies of MHD, sound and Brunt-Väisälä frequencies, respectively; $V_{a}^{2}=H_{0}^{2} / 4 \pi \bar{\rho}$ is the square of the velocity of MHD waves, $k=\sqrt{k_{\perp}^{2}+m^{2}}-$ total wavenumber, $k_{\perp}^{2}=k_{x}^{2}+k_{y}^{2}, m=k_{z}-i / 2 H$. The introduction of complex $m$ formally reduces the problem of wave propagation in an inhomogeneous atmosphere to the case of homogeneous medium.

The first bracket describes propagation of transverse Alfvén's waves in the ionosphere. Compressibility and stratification of the ionosphere do not play any role in the Alfvén's waves and, therefore, for these waves the transversality condition $(\boldsymbol{k} \times \boldsymbol{V})=0$ is always satisfied. In this case, an imaginary term can be neglected in the expression for the wavenumber $m$ (since in the absence of stratification $g=0$ and $H=\infty)$. As a result in the Alfvén's frequency $\omega_{a}=V_{a} k$ total wavenumber is a real number. Square bracket in Eq. (3) describes propagation of magnetoacoustic and magneto-gravity waves in the ionosphere. It contains important special cases: a) in the absence of magnetic field $\left(V_{a}=0\right)$ the expression in square brackets passes into the well-known dispersion equation for the acoustic-gravity waves (AGW) (Prandtl, 1952):

$\omega^{4}-\omega^{2}\left(\omega_{\mathrm{s}}^{2}+i \frac{\omega_{\mathrm{s}}^{2}}{k H} \cos \theta_{k}\right)+N^{2} \omega_{\mathrm{s}}^{2} \sin ^{2} \theta_{k}=0$.

Getting rid of imaginary terms, we can easily obtain standard dispersion equation for AGW (Khantadze, 1973):

$\frac{\omega^{2}}{\omega_{a c}^{2}}+\frac{\omega_{g}^{2}}{\omega^{2}}=1$,

$\omega_{\mathrm{ac}}^{2}=\mathfrak{x} H\left(k_{x}^{2}+k_{y}^{2}+k_{z}^{2}+1 / 4 H^{2}\right)$,

$\omega_{g}^{2}=\frac{g}{H}\left(1-\mathfrak{x}^{-1}\right) \frac{k_{x}^{2}+k_{y}^{2}}{k_{x}^{2}+k_{y}^{2}+k_{z}^{2}+1 / 4 H^{2}}$.

Let's carry out a short analysis of the waves under consideration.

1) For the incompressible ionosphere in the polytrophic equation $\rho P^{-1 / \mathfrak{x}}=$ const the value $\mathfrak{x}=\infty$ should be placed, which is the unique condition when density remains constant. As it follows from Eq. (6), if $\mathfrak{x}=\infty$, all frequencies, corresponding to the acoustic waves $\omega_{a c}$, become infinite, i.e. waves with these frequencies vanish since the phase velocities of these waves tend to infinity and their periods tend to zero. Thus, in the incompressible medium, only oscillations with frequencies $\omega_{g}$ can arise at $æ \rightarrow \infty$ :

$\omega_{g}^{2}=\frac{g}{H} \frac{k_{\perp}^{2}}{k_{\perp}^{2}+k_{z}^{2}+1 / 4 H^{2}}$.

Waves having these frequencies are called the internal gravity waves. Thus, compressibility is a decisive factor for the existence of acoustic waves.

2) If $æ \rightarrow 1$ in the polytrophic equation $P^{1-(1 / \mathfrak{x})} / T=$ const temperature will be conserved quantity since this case corresponds to isothermal processes with respect to which stratification of the medium does not play any role. Isothermally displaced along the vertical particle, it has the same temperature as the surrounding particles and does not experience any buoyancy forces from them. If $\mathfrak{x} \rightarrow 1$ all frequencies $\omega_{g}$ vanish, so oscillations with these frequencies disappear. Thus, in the isothermal medium only oscillations with $\omega_{\mathrm{ac}}$ frequencies:

$\omega_{\mathrm{ac}}^{2}=g H\left(k_{\perp}^{2}+k_{z}^{2}+1 / 4 H^{2}\right)$,

can occur, which are defined at $æ \rightarrow 1$. Waves with these frequencies are called internal acoustic waves. Thus, unlike acoustic waves, stable stratification or Archimedean buoyancy can be considered as the main reason of gravitational oscillations. Since under the real conditions in the atmosphere propagate both acoustic and gravitational waves, it is 
assumed that wavy processes run adiabatically $(\mathfrak{x}=\gamma=1.4)$ as, in this case density, does not remain constant $(\mathfrak{x} \neq 0)$ and wavy motions with frequencies $\omega_{a c}$ remain. On the other hand, the condition of isothermal motion is not satisfied $(\mathfrak{x} \neq 1)$ as well. Consequently, wavy motions with frequencies $\omega_{g}$ also remain. From Eq. (5) it follows that the gravitational waves are also always more low-frequency than acoustic waves. In the ionospheric F-region, acoustic waves have a period of 5-13 min and gravitational 1-1.5 $\mathrm{h}$. The maximum velocity of the AGW propagation in the ionosphere does not exceed 700-800 $\mathrm{m} \mathrm{s}^{-1}$ (Khantadze, 1973; Grigor'ev, 1999). As it follows from Eq. (6), AGW always have a vertical component, i.e. they are essentially three-dimensional and, therefore, they were called internal waves (Monin and Obukhov, 1958). Nonlinear acoustic-gravity waves were considered by Stenflo and Shukla (2009).

b) In the absence of stratification $(g=0)$, when the perturbed pressure is a function of only perturbed density, the square bracket in Eq. (3) gives the dispersion equation for the fast and slow magneto-acoustic waves (Kulsrud, 2005):

$\omega^{4}-\omega^{2}\left(c_{\mathrm{s}}^{2}+V_{a}^{2}\right) k^{2}+V_{a}^{2} c_{\mathrm{s}}^{2} k^{4} \cos ^{2} \theta_{k H}=0$.

Here, the wave number $k$ is real. Solving Eq. (9) with respect to the phase velocity $c_{\mathrm{ph}}=\omega / k$, we obtain:

$$
\begin{aligned}
c_{\mathrm{ph} \pm}= & \frac{1}{2}\left(\sqrt{c_{\mathrm{s}}^{2}+V_{a}^{2}+2 c_{\mathrm{s}} V_{a} \cos \theta_{k H}}\right. \\
& \left. \pm \sqrt{c_{\mathrm{s}}^{2}+V_{a}^{2}-2 c_{\mathrm{s}} V_{a} \cos \theta_{k H}}\right) .
\end{aligned}
$$

The sign (+) corresponds to the fast magneto-acoustic waves and $(-)$ to the slow magneto-acoustic waves. In addition, waves propagating both in positive and negative directions correspond to each $c_{\mathrm{ph}+}$ and $c_{\mathrm{ph}-}$.

Cross and dot multiplication of the linearized momentum equation by wave-vector $\boldsymbol{k}$ (at $\boldsymbol{g}=0, \omega_{\mathbf{0}}=0$ ) gives:

$$
\boldsymbol{V} \times \boldsymbol{k}=-\frac{\left(\boldsymbol{k} \times \boldsymbol{H}_{\mathbf{0}}\right)}{4 \pi \rho} \boldsymbol{h} \times \boldsymbol{k}, \quad \bar{\rho}(\boldsymbol{V} \times \boldsymbol{k})=P+\frac{\left(\boldsymbol{H}_{\mathbf{0}} \times \boldsymbol{h}\right)}{4 \pi} .
$$

Here $\boldsymbol{h}$ is the perturbation of the geomagnetic field.

From Eq. (11) it follows that fast and slow magnetoacoustic waves in the ionosphere, under the considered approximation, have neither a longitudinal $(\boldsymbol{V} \times \boldsymbol{k}=0)$ nor a transverse $((\boldsymbol{V} \times \boldsymbol{k})=0)$ nature, i.e. we have waves of mixed type. From Eq. (10) follows that depending on an angle $\theta_{k H}$, the nature of slow magneto-acoustic waves will significantly change. Indeed, if $\theta_{k H}=0$, i.e. $\boldsymbol{k} \| \boldsymbol{H}_{\mathbf{0}}$, from Eq. (10) we get $c_{\mathrm{ph}+}=c_{\mathrm{s}}$ and $c_{\mathrm{ph}-}=V_{a}$. Similar to this case, if $\theta_{k H}=\pi / 2$, i.e. when $\boldsymbol{k} \perp \boldsymbol{H}_{\mathbf{0}}$, we get $c_{\mathrm{ph}+}=\sqrt{c_{\mathrm{s}}^{2}+V_{a}^{2}}, c_{\mathrm{ph}-}=0$. In the case of other angles of $\theta_{k H}$, the proposed (Khantadze, 1973) graphical method by Friedrichs is usually used. For the evaluation of the angle $\alpha$ between the velocity vector $\boldsymbol{V}$ and wave vector $\boldsymbol{k}$, we easily get from expression (11):

$\operatorname{tg} \alpha=\frac{\sin \theta_{k H} \cdot \cos \theta_{k H}}{\cos ^{2} \theta_{k H}-c_{\mathrm{ph}}^{2} / V_{a}^{2}}$.
Since the value $\alpha$ differs from 0 and $\pi / 2$ (except when $\theta_{k H}$ is equal to 0 or $\pi / 2)$ in the general case magneto-acoustic waves actually are neither purely longitudinal $(\alpha=0)$ nor purely transverse $(\alpha=\pi / 2)$. At $\theta_{k H}=0$ slow magnetoacoustic wave $c_{\mathrm{ph}-}$ is transverse $(\alpha=\pi / 2)$ and propagates in parallels to the magnetic field $\boldsymbol{H}_{\mathbf{0}}$ with velocity $\boldsymbol{V}_{a}$. In other words, it degenerates into an ordinary MHD wave.

Similarly, at $\theta_{k H}=0$ the fast magneto-sonic wave is longitudinal $(\alpha=0)$ and propagates in parallels with $\boldsymbol{H}_{\mathbf{0}}$ at the speed of sound $c_{\mathrm{s}}$. From Eq. (10) it also follows that at $\theta_{k H}=\pi / 2$ longitudinal waves $(\alpha=0)$ exist, which propagate with the velocity $\sqrt{c_{\mathrm{s}}^{2}+V_{a}^{2}}$.

Without going into the more details on the separate consideration of the internal and MHD waves, the study of coexistence between these waves in the ionosphere is of real interest. Direct analysis of the joint consideration of AGW and MHD waves on the basis of the fundamental dispersion Eq. (3) is quite difficult and, therefore, in the present paper, starting from the specificity of dynamic processes in the ionosphere, we restrict ourselves to some special cases of interest for the ionospheric physics. Interest in these cases is caused by the fact that at heights starting from $130 \mathrm{~km}$ and higher, the influence of the geomagnetic field on the wave processes dominates over the neutral pressures in the ionospheric plasma and correspondingly the role of electromagnetic processes in the ionosphere is increasing. Presence of the electric conductivity and geomagnetic field $\boldsymbol{H}_{\mathbf{0}}$ gives to the upper atmosphere additional elasticity of an electromagnetic nature. In particular, in this case, the nature of the acting forces in the magnetic field on the atmospheric particle (expressed by Maxwell stress tensor) is such that magnetic lines of force during the interaction with medium lengthen (shorten) and at the same time attract and repel each other.

Thus, above $130 \mathrm{~km}$, the electromagnetic elasticity of the geomagnetic field in the ionosphere must generate transverse MHD waves of Alfvén type and longitudinal waves - "magnetic" sound as well. In the lower E-region $(70-130 \mathrm{~km})$, called Hall's region $(\delta=1)$, besides the electromagnetic elasticity hydrodynamic elasticity of the ionosphere also becomes important, which is caused by compressibility, stratification and rotation of the Earth. To get rid of hydrodynamic effects, let us take into account the ionospheric levels, according to the numerous observations (Sorokin and Federovich, 1982; Sharadze, 1991), at the ionospheric moderate and high latitudes large-scale (up to $10^{3} \mathrm{~km}$ ), and longperiod (with a characteristic time scale $0.5-2 \mathrm{~h}$ ) ionospheric wavy perturbations regularly exist which are propagating zonally over long distances (to tens of thousands of kms) with the velocity more that $1 \mathrm{~km} \mathrm{~s}^{-1}$.

The observed propagation velocity of wave can not be explained within the frames of hydrodynamic theory of ordinary AGW, since the maximum characteristic velocity of the letter, as it was noted above, at the ionosphere altitudes does not exceed $700-800 \mathrm{~m} \mathrm{~s}^{-1}$. Let us show that the velocities of the order of $1 \mathrm{~km} \mathrm{~s}^{-1}$ and more are arising when the influence 
of partial "freezing-in" of the geomagnetic field on the propagation of MHD waves in the ionosphere is taking into account.

Though in the neutral component of the ionosphere, the velocities of the order of $1 \mathrm{~km} \mathrm{~s}^{-1}$ and more are large (supersonic), they are insignificant for MHD waves in the plasma component $\left(\sim 10^{3} \mathrm{~km} \mathrm{~s}^{-1}\right)$. This is stipulated by the fact that in the ionosphere for the long-period processes the geomagnetic field is "frozen" into the plasma component (Khantadze, 1973) and during the perturbations it passes its perturbation to the neutral component by collision processes. Further in the neutral part, it propagates with the velocity $V_{a}=H_{0} / \sqrt{4 \pi \rho}=H_{0} / \sqrt{4 \pi M N_{n}}=\sqrt{\eta} V_{A}$, where $\eta=N /\left(N_{n}+N\right)$ is the ionization degree of the ionospheric medium, $V_{A}=H_{0} / \sqrt{4 \pi M N}$ is the velocity of the MHD wave in the plasma component of ionosphere. In the ionospheric $E(70-150 \mathrm{~km})$ and $F(150-600 \mathrm{~km})$ regions $\eta=$ $N / N_{n} \sim\left(10^{-8} \div 10^{-4}\right) \ll 1$ and, therefore, the value of $V_{a}$ is much less than $V_{A}$. Consequently, we naturally come to the consideration of slow (in the electrodynamics sense) longperiod MHD waves in the ionosphere.

\section{Slow MHD waves in the ionosphere}

Propagating MHD waves gather information about the magnetosphere, ionosphere and the ground. The ionospheric observations reveal one more class of the electromagnetic perturbations in E- and F-regions, known as the slow MHD waves (Kamide and Baumjohan, 1993; Sorokin and Fedorovich, 1982). These waves are insensitive to spatial inhomogeneities of the Coriolis and Ampere forces and propagate in the ionospheric medium more slowly than the ordinary MHD waves. In natural conditions, these perturbations are revealed as background oscillations. The forced oscillations of this kind are generated by an impulse action on the ionosphere from above, during magnetic storms (Hajkowicz, 1991; Lysak et al., 2009), or from below, as a result of earthquakes, volcanic eruption or artificial explosions (Pokhotelov et al., 1995, 2001; Shaefer et al., 1999). In the last case, the perturbations are revealed as solitary vortex structures.

To separate electromagnetic effects of slow MHD waves, we neglect all hydrodynamic forces in the set of momentum Eq. (1). As a result we get:

$$
\frac{d \boldsymbol{V}}{d t}=\frac{1}{4 \pi M N_{n}} \nabla \times \boldsymbol{H} \times \boldsymbol{H}=\frac{1}{\rho c} \boldsymbol{j} \times \boldsymbol{H}_{\mathbf{0}},
$$

where $\boldsymbol{j}$ is the current density, and $\rho=\rho_{n}+\rho_{p l} \approx \rho_{n}=$ $M N_{n}$.

We investigate MHD waves separately for the ionospheric E- and F-regions. For the E-region the plasma component behaves like a passive impurity. The neutrals completely drag ions and the "ionospheric" friction between neutrals and ions can be neglected (Kelley, 1989).
Generalized Ohm's law for the E-region can be expressed in the following form (Khantadze, 1973; Sorokin and Fedorovich, 1982)

$$
\frac{1}{c} \boldsymbol{j} \times \boldsymbol{H}=e N\left(\boldsymbol{E}+\frac{1}{c} \boldsymbol{V} \times \boldsymbol{H}\right) .
$$

Using Maxwell's equation $\partial \boldsymbol{H} / \partial t=-c \nabla \times \boldsymbol{E}$ and excluding $\boldsymbol{E}$ with the help of Eq. (14) and Ampere force $\boldsymbol{F}_{\boldsymbol{A}}=$ $\boldsymbol{j} \times \boldsymbol{H} / c$ from Eq. (13), we get an induction equation for the magnetic field:

$\frac{\partial \boldsymbol{H}}{\partial t}=\nabla \times \boldsymbol{V} \times \boldsymbol{H}-\frac{N_{n}}{N} \frac{M c}{e} \nabla \times \frac{d \boldsymbol{V}}{d t}$.

Equation (15) completely coincides with the third equation of the system (1), where the last term appears because of the Hall's effect and leads to the dispersion of MHD waves when $\delta=1$.

Using the Fridman designations, Eq. (15) can be rewritten as:

$\operatorname{helm}\left(\nabla \times \boldsymbol{V}+\frac{N}{N_{n}} \frac{e}{M c} \boldsymbol{H}_{\mathbf{0}}\right)=0$,

where $\boldsymbol{H}=\boldsymbol{H}_{0}+\boldsymbol{h} \approx \boldsymbol{H}_{0}$. Operator helm, introduced by Fridman in honour of Helmholtz for any vector field $\boldsymbol{a}$, has the following form:

helma $=\frac{\partial \boldsymbol{a}}{\partial t}-\nabla \times \boldsymbol{V} \times \boldsymbol{a}+\boldsymbol{a} \nabla \cdot \boldsymbol{V}$.

Fridman showed that the equality helma $=0$ means conservation (freezing-in) of both vector lines and intensity of vector tubes of vector $\boldsymbol{a}$, i.e. when helma $\boldsymbol{a}=0$ vector $\boldsymbol{a}$ is invariant (Fridman, 1934). First found by Khantadze (1973) was the invariant $\nabla \times \boldsymbol{V}+2 \boldsymbol{\omega}_{0}+\mathrm{Ne} \boldsymbol{H}_{0} / N_{n} M c$ for the ionospheric medium.

From the expression (16) two important consequences follow:

- In the ionospheric E-region, the condition of freezingin of the geomagnetic field $\boldsymbol{H}_{\mathbf{0}}$ is not fulfilled, however, the vector $\nabla \times \boldsymbol{V}+\eta \boldsymbol{\omega}_{\boldsymbol{i}}$ (where $\boldsymbol{\omega}_{\boldsymbol{i}}=e \boldsymbol{H}_{\mathbf{0}} / M c$ is the cyclotron frequency of ions) is frozen in the medium. In addition the deviation from freezing-in is determined by the magnitude of neutral vortex $\nabla \times \boldsymbol{V}$ in the E-region of ionosphere. In case of the vortex-free motion $(\nabla \times \boldsymbol{V}=$ $0)$, the geomagnetic field at the altitudes of E-region will be completely frozen-in (helm $\left.\boldsymbol{H}_{\mathbf{0}}=0\right)$ ).

- According to Eq. (16), the geomagnetic field (as the angular velocity of the Earth's rotation $\omega_{0}$ ) must generate large-scale vortex $\boldsymbol{\Omega}=\nabla \times \boldsymbol{V}$ in the E-region, since at these altitudes $N \omega_{i} / N_{n}$ (as $2 \omega_{0}$ in the "modified" vortex $\left.\operatorname{helm}\left(\boldsymbol{\Omega}+2 \boldsymbol{\omega}_{\mathbf{0}}\right)\right)$ is of the order of $\sim 10^{-4} \mathrm{~s}^{-1}$.

Linearizing Eqs. (13) and (14), and limiting ourselves to the moderate and high latitudes, by means of the traditional method dispersion equation for the plane MHD waves, can 
be obtained. However, to clearly demonstrate how the wave equation looks like in this case, we will act differently. Solving Eq. (14) and linearized Eq. (13) with respect to $\boldsymbol{E}$ and $\boldsymbol{j}$ and assuming that the equality $\left(\boldsymbol{j} \times \boldsymbol{H}_{\mathbf{0}}\right)=0$ (Sorokin and Fedorovich, 1982) is fulfilled, we get:

$\boldsymbol{E}=-\boldsymbol{w}-i \frac{\omega}{\Omega_{i}} \boldsymbol{w} \times \boldsymbol{\tau}, \quad$ and $\quad \boldsymbol{j}=\frac{\rho c^{2}}{H_{0}^{2}} \frac{\partial \boldsymbol{w}}{\partial t}$,

where $\boldsymbol{w}=\boldsymbol{V} \times \boldsymbol{H}_{\mathbf{0}} / c$ is the dynamo field, $\omega$ is the frequency of wavy perturbation, $\rho=M N_{n}, \boldsymbol{\tau}=\boldsymbol{H}_{\mathbf{0}} / H_{0}, \Omega_{i}=\eta \omega_{i}$ is modified by the ionization degree cyclotron frequency of ions, and $\eta=N / N_{n}$.

Substituting Eq. (17) into Maxwell's equation $\nabla \times \nabla \times$ $\boldsymbol{E}=-\left(4 \pi / c^{2}\right) \partial \boldsymbol{j} / \partial t$, we obtain the wave equation:

$\frac{\partial^{2} \boldsymbol{w}}{\partial t^{2}}-V_{a}^{2} \nabla \times \nabla \times \boldsymbol{w}=i \frac{\omega}{\Omega_{i}} V_{a}^{2} \nabla \times \nabla \times \boldsymbol{w} \times \boldsymbol{\tau}$.

Here the last term takes into account the Hall's effect. At $w \sim \exp \left(-i \omega t+i k_{x} x+i k_{z} z\right)$ from Eq. (18) the following dispersion equation is easily obtained:

$\left(\omega^{2}-V_{a}^{2} k_{x}^{2}\right)\left(\omega^{2}-V_{a}^{2} k_{z}^{2}\right)=\frac{\omega^{2} k_{x}^{2} k_{z}^{2} V_{a}^{4}}{\Omega_{i}^{2}}$.

Equation (19) (in the electrodynamics sense) describes very slow, long-period MHD waves in the ionospheric Eregion. For pure plasma $(\eta=1)$ MHD waves become high-frequency and small-scale (Kadomtsev, 1982). From Eq. (19) at almost transversal propagation with respect to $\boldsymbol{H}_{\mathbf{0}} \approx H_{0 z} \boldsymbol{e}_{z}$, when $k_{z}^{2} \ll k_{x}^{2}$ and $\omega \gg V_{a} k_{z}$, for magnetoacoustic waves we get:

$\omega_{\mathrm{ma}}^{2}=V_{a}^{2} k_{x}^{2}\left(1+\frac{\theta^{2} k_{x}^{2} V_{a}^{2}}{\Omega_{i}^{2}}\right), \quad \theta=\frac{k_{x}}{k_{z}}$.

From Eq. (20) it follows that in the E-region the characteristic horizontal wavelength $\lambda_{0}=\left(2 \pi \theta V_{a}\right) / \Omega_{i}$ exists, which determines the characteristic "length of dispersion" caused by the Hall's effect. At $\lambda_{x}>\lambda_{0}$ magneto-acoustic wave undergoes weak dispersion, and at $\lambda_{x}<\lambda_{0}$ the dispersion is strong. From Eq. (20) it also follows that at a small $k_{x}$ frequency of the magneto-acoustic $\omega_{m a}=V_{a} k_{x}$ increases linearly with $k_{x}$. At large $k_{x}$ (when the unit in brackets Eq. (20) can be neglected), wave frequency is subject to the frequency of helicons $\omega_{h}$ :

$\omega=\omega_{h}=\frac{c k_{x} k_{z}}{4 \pi e N} H_{0 z}$.

In ionospheric physics, they are known as "atmospheric whistlers". As a result, helicons in the E-region are the limiting case of magnetic sound. In helicons only electrons of the ionospheric plasma are oscillating together with the frozen in geomagnetic field lines.
For the second root, taking into account Eq. (19), at $k_{z}^{2} \ll$ $k_{x}^{2}$ and $\omega^{2} \ll V_{a}^{2} k_{x}^{2}$ we get:

$$
\begin{aligned}
\omega^{2} & =\omega_{a}^{2}=V_{a}^{2} k_{z}^{2} \frac{\Omega_{i}^{2}}{\Omega_{i}^{2}+V_{a}^{2} k_{z}^{2}} \\
& =V_{a}^{2} k^{2} \frac{\Omega_{i}^{2}}{\Omega_{i}^{2}+V_{a}^{2} k_{z}^{2}} \cos ^{2} \theta_{k} H_{0},
\end{aligned}
$$

where $k=\sqrt{k_{x}^{2}+k_{z}^{2}}, \theta_{k H_{0}}$ is the angle between $\boldsymbol{k}$ and $H_{0 z} \boldsymbol{e}_{z}$. Expression (22) describes Alfvén wave with dispersion. At $k_{z} \rightarrow \infty$ the wave frequency is subject to the characteristic frequency $\omega \rightarrow \Omega_{i}=\eta \omega_{i}$ (in the E-region $\Omega_{i}$ is of the order of $10^{-4}-10^{-5} \mathrm{~s}^{-1}$ ). Consequently, waves $\Omega_{i}$ in the ionosphere are the limiting case of the quasi-transversal very lowfrequency Alfvén waves. From Eq. (22) follows also that Alfvén waves in the ionosphere can be very low-frequency when the angle $\theta_{k H_{0}}$ is subject to $\pi / 2$.

In the F-region (where Hall's effect is absent), the ionospheric medium becomes strongly dissipative, since in this region of the upper atmosphere ions-neutrals drag does not occur (Kelley, 1989). Wave equation for the F-region has the following form:

$\frac{\partial^{2} \boldsymbol{w}}{\partial t^{2}}-V_{a}^{2} \nabla \times \nabla \times \boldsymbol{w}=-i \frac{\omega}{v_{i}} V_{a}^{2} \nabla \times \nabla \times \boldsymbol{w}$,

where $v_{i}=\eta v_{\text {in }}, v_{\text {in }}$ is the collision frequency of ions with neutrals (in the F-region $v_{i} \sim 10^{-3} \mathrm{~s}^{-1}$ ).

At $\omega / v_{i} \ll 1$, Eq. (23) describes magneto-acoustic and Alfvén waves in the F-region. In the reverse limiting case $\omega / v_{i} \gg 1$ from Eq. (23) we obtain the diffusion type equation (Khantadze, 1973):

$\frac{\partial \boldsymbol{w}}{\partial t}=D \nabla \times \nabla \times \boldsymbol{w}$,

where $D=V_{a}^{2} / \nu_{i}$ is the diffusion coefficient of the medium in the ionospheric F-region. In this case, dynamo field $\boldsymbol{w}$ is damping and the solution takes the form of the temperature wave. Consequently, in the general case of Eq. (23) for the F-region describes the propagation of magneto-acoustic and Alfvén waves with damping.

The expressions (20) and (22) allow us to estimate the periods and velocities at quasi-transversal propagation of the waves under consideration. For example, for the maximum wavelength $\lambda_{x}=10^{3} \mathrm{~km}, \theta=10^{-2}, \Omega_{i}=5 \times 10^{-5} \mathrm{~s}^{-1}$, from Eqs. (20) and (22), we obtain the approximate expressions for the frequencies of MHD waves in the E-region:

$\omega_{\mathrm{ma}}^{2}=\frac{\Omega_{i}^{2}}{\theta^{2}}\left(\frac{\lambda_{0}}{\lambda_{x}}\right)^{4}, \quad \omega_{a}^{2}=\Omega_{i}^{2}\left(1-\frac{\lambda_{x}^{2}}{\lambda_{0}^{2}}\right)$,

and it follows that $T_{\mathrm{ms}} \approx 20 \mathrm{~min}, c_{\mathrm{ma}}=1 \mathrm{~km} \mathrm{~s}^{-1}, T_{a} \approx 2$ days, $c_{a}=10 \mathrm{~m} \mathrm{~s}^{-1}(c=\omega / k$ is the phase velocity).

Thus, the above-mentioned observed perturbations can be identified with magneto-acoustic waves with frequency 
(Eq. 20). Alfvén type waves, as it is seen from Eq. (25), are very slow and long-period like planetary waves in the ionospheric E-region. This feature of the Alfvén waves (which follows from Eq. (22) at $\theta_{k H_{0}} \rightarrow \pi / 2$ ) can play an important role in the generation of low-frequency electromagnetic planetary waves in high-latitude ionosphere (Khantadze et al., 1982). Alfvén waves can be very slow and low-frequency in the F-region as well at quasi-transversal propagation. Propagation of slow MHD waves is examined in more detail in (Sorokin and Fedorovich, 1982).

\section{The influence of geomagnetic field on propagation of AGW in the ionosphere}

The importance of gravity waves for the dynamics of the middle atmosphere has been recognised for quite a while (Hines, 1960; Hooke, 1968). As they propagate upward mainly from tropospheric sources, their amplitude grows due to the vertical ambient density gradient (Grigor'ev, 1999). Unless they are absorbed at some critical layer or get deflected by wind or stratification gradients, they eventually become subject to wave breaking (Fritts and Alexander, 2003). The associated momentum and energy deposition is essential for the understanding of the middle atmosphere's general circulation (Houghton, 1978; Lindzen, 1981; Holton, 1982, 1983; Garcia and Solomon, 1985). Another effect might be the generation of turbulence by gravity wave breaking, thus, leading to frictional heating rates of relevance for the mesosphere energy balance (Lübken, 1997; Becker and Schmitz, 2002; Müllemann et al., 2003).

In the partially ionized ionospheric E-layer, the electromagnetic inertio-gravity (IG) waves were investigated by Kaladze et al. (2007). It was shown that the free energy necessary for linear instability of electromagnetic IG waves arises from the field-aligned current. For the ionospheric generalization of tropospheric AGW the propagation of so called "magnetized acoustic-gravity waves" (MAGW) in the conductive ionosphere was considered by Kaladze et al. (2008). Such waves do not significantly perturb the geomagnetic field and are solely excited by the ionospheric dynamo electric field. The incorporation of the Coriolis force leads to the coupling of AGW with the inertial waves. This results in the appearance of inertio-acoustic-gravity (IAG) waves and the additional cut-off frequency at $2 \omega_{0}$ appears. It was shown that, for the ionospheric E-layer, the influence of the electromagnetic force is essential (the Hall conductivity predominates) along with the Coriolis force and they provide opposite effects. As to the ionospheric F-layer, the action of the Ampere force is defined by the Pedersen conductivity which leads to the damping of MAGW.

For the influential investigation of the geomagnetic field on the propagation of AGW in the ionosphere, we will follow the classical fundamental paper by Monin-Obukhov (1958), which completely describes all properties of AGW in the troposphere. In this paper, the role of the Earth's rotation in propagation of acoustic-gravity waves was first shown by means of the Coriolis parameter $\ell=2 \omega_{0 z}=2 \omega_{0} \cos \theta$, where $\theta=\pi / 2-\chi$ is the co-latitude of location. Shown below, will be another fundamental parameter of the Earth; a vertical component of the geomagnetic field $H_{0 z}=-2 H_{E} \cos \theta$, ( $H_{E}$ is the value of the geomagnetic field at the equator), that significantly affects the nature of propagation of acousticgravity waves in the ionosphere.

We neglect, for simplicity, the action of the Coriolis force and linearize the system (1) for the ionospheric F-region ( $\delta=$ $0)$ with respect to the equilibrium state. We restrict ourselves by moderate and high latitudes $\left(\boldsymbol{H}_{\mathbf{0}} \approx H_{0 z} \boldsymbol{e}_{z}\right)$, neglecting the action of the ordinary Obukhov parameter $L_{0}=c_{\mathrm{s}} / l$. Furthermore, we introduce the new variables for the fluxes $\bar{\rho} V_{x}$, $\bar{\rho} V_{y}$, and components of the induced magnetic field $h_{x}, h_{y}$ :

$$
\begin{aligned}
& \bar{\rho} V_{x}=\frac{\partial \varphi}{\partial x}-\frac{\partial \psi}{\partial y}, \quad \bar{\rho} V_{y}=\frac{\partial \varphi}{\partial y}+\frac{\partial \psi}{\partial x}, \\
& h_{x}=\frac{\partial \varphi_{a}}{\partial x}-\frac{\partial \psi_{a}}{\partial y} \quad, \quad h_{y}=\frac{\partial \varphi_{a}}{\partial y}+\frac{\partial \psi_{a}}{\partial x},
\end{aligned}
$$

requiring regularity of the functions $\psi, \psi_{a}, \varphi, \varphi_{a}$ at infinity. Then for small- and medium-scale perturbations, we easily obtain the generalized set of Monin-Obukhov wave equations for the ionospheric F-region (Monin and Obukhov, 1958):

$$
\begin{aligned}
& \frac{\partial \psi}{\partial t}=\frac{H_{0 z}}{4 \pi} \frac{\partial \psi_{a}}{\partial z}, \\
& \frac{\partial \varphi}{\partial t}=-P-\frac{H_{0 z}}{4 \pi} h_{z}+\frac{H_{0 z}}{4 \pi} \frac{\partial \varphi_{a}}{\partial z}, \\
& \frac{\partial P}{\partial t}=-c_{\mathrm{s}} \Delta \varphi-\beta f-c_{s}^{2} \frac{\partial f}{\partial z}, \\
& \frac{\partial \rho}{\partial t}=-\Delta \varphi-\frac{\partial f}{\partial z}, \quad, \quad \frac{\partial \psi_{a}}{\partial t}=H_{0 z} \frac{1}{\bar{\rho}} \frac{\partial \psi}{\partial z}, \\
& \frac{\partial f}{\partial t}=-\left(\frac{\partial P}{\partial z}+\rho g\right), \quad \frac{\partial h_{z}}{\partial t}=-\frac{H_{0 z}}{\bar{\rho}} \Delta \varphi, \quad \frac{\partial h_{z}}{\partial z}=-\Delta \varphi_{a} . \\
& \frac{\partial \varphi_{a}}{\partial t}=\frac{H_{0 z}}{\bar{\rho}} \frac{\partial \varphi}{\partial z},,
\end{aligned}
$$

Here $c_{\mathrm{s}}^{2}=\mathfrak{P} / \bar{\rho}$ is the squared speed of sound, $\bar{\rho}-$ density of medium in the unperturbed state depending only on the height $z$ and connecting with unperturbed pressure by the static equation $\partial \bar{P} / \partial z=-\bar{\rho} g, f=\bar{\rho} V_{z}$ is the vertical airflow, $\beta=(æ-1) g$ - parameter of thermal stability of the atmosphere, $\Delta=\partial^{2} / \partial x^{2}+\partial^{2} / \partial y^{2}$ - two-dimensional Laplace operator. In the absence of the magnetic field, the system (28) coincides with the Monin-Obukhov (1958) set of equations.

Equations for $\psi$ and $\psi_{a}$ create the closed system and we get

$\frac{\partial^{2} \psi}{\partial t^{2}}-\frac{\partial}{\partial z} V_{a}^{2} \frac{\partial \psi}{\partial z}=0$ 
Here $V_{a}^{2}=H_{0 z}^{2} / 4 \pi \bar{\rho}$ is the squared Alfvén velocity of MHD waves. Assuming the sound and Alfvén velocities are constant, we get the Alfvén wave equation

$$
\frac{\partial^{2} \psi}{\partial t^{2}}-V_{a}^{2} \frac{\partial^{2} \psi}{\partial z^{2}}=0
$$

This equation, as Eq. (3), describes the propagation of transverse Alfvén waves in the ionosphere (Kadomtsev, 1982) with the phase velocity corresponding to the velocity of the slow MHD wave $\boldsymbol{V}_{a}$. In the ionosphere, Alfvén waves always propagate only along force lines of the geomagnetic field $\boldsymbol{H}_{0}$. As it was noted above, the thermodynamic parameters of medium, pressure, density and temperature are not perturbed when such transverse electromagnetic waves are propagating in the ionosphere (Khantadze, 1973). The source of such waves is the tension of field lines of the geomagnetic field $\boldsymbol{H}_{0}$.

At the variable $V_{a}^{2}$, when $\bar{\rho}=\rho_{0} \exp (-z / H)$ Eq. (29) can be solved through the special functions. According to Yaglom (1953), we may get rid of the height dependence of the parameters of the system (28) if we average all variables over height in the semi-space $(0, \infty)$. But taking into account that our paper has the review character, we confine ourselves with the rougher approximation of the "frozen in" coefficients in the system (28).

In this case for the variables $\varphi$ and $\bar{\rho} V_{z}$, we get the wave equations

$$
\begin{aligned}
& \frac{\partial^{2} \varphi}{\partial t^{2}}=\left(c_{\mathrm{s}}^{2}+V_{a}^{2}\right) \Delta \varphi+V_{a}^{2} \frac{\partial^{2} \varphi}{\partial z^{2}}+\beta f+c_{\mathrm{s}}^{2} \frac{\partial f}{\partial z} \\
& \frac{\partial^{2} f}{\partial t^{2}}=\frac{\partial}{\partial z}\left(\beta f+c_{s}^{2} \frac{\partial f}{\partial z}+c_{\mathrm{s}}^{2} \Delta \varphi\right)+g\left(\frac{\partial f}{\partial z}+\Delta \varphi\right) .
\end{aligned}
$$

We search the solutions of Eqs. (31) and (32) in the form of harmonic waves with the amplitudes depending on $z$, i.e.

$$
\begin{aligned}
& \varphi(x, y, z, t)=\phi(z) \exp \left[i\left(k_{x} x+k_{y} y-\omega t\right)\right], \\
& f(x, y, z, t)=\mathrm{X}(z) \exp \left[i\left(k_{x} x+k_{y} y-\omega t\right)\right],
\end{aligned}
$$

where $k_{x}, k_{y}$ are arbitrary horizontal wavenumbers, and $\omega$ are frequencies to be determined. We get the following equations for amplitudes $\phi(z)$ and $\mathrm{X}(z)$ :

$\left(l_{H}^{2}+k^{2} c_{\mathrm{s}}^{2}-\omega^{2}\right) \Phi=\beta X+c_{\mathrm{s}}^{2} \frac{d X}{d z}$,

$\left(l_{H}^{2}-\omega^{2}\right)\left(c_{\mathrm{s}}^{2} \frac{d \Phi}{d z}+g \Phi\right)=\left(\beta g-c_{\mathrm{s}}^{2} \omega^{2}\right) ?$.

Here $k^{2}=k_{x}^{2}+k_{y}^{2}, l_{H}^{2}=k^{2} V_{a}^{2}-V_{a}^{2} d^{2} / d z^{2}$ is the differential operator. Equations (34) and (35) exactly coincide with the Monin-Obukhov equations (1958) if, instead of the operator $l_{H}$, the Coriolis parameter $l=2 \omega_{0 z}$ will be substituted. Equations (34) and (35) have nontrivial partial solutions, where $X=0$. Indeed, in this case

$$
\left(l_{H}^{2}+k^{2} c_{s}^{2}-\omega^{2}\right) \Phi=0,
$$

$$
\left(l_{H}^{2}-\omega^{2}\right)\left(c_{\mathrm{s}}^{2} \frac{d \Phi}{d z}+g \Phi\right)=0 .
$$

If we exclude the trivial case $\Phi=0$ from Eqs. (36) and (37), we find that $l_{H}^{2}-\omega^{2} \neq 0$. Then the amplitude $\Phi(z)$ must satisfy the equation

$c_{\mathrm{s}}^{2} \frac{d \Phi}{d z}+g \Phi=0$,

and the solution has the following form:

$\phi(z)=\phi_{0} \exp \left[-g\left(z-z_{0}\right) / c_{s}^{2}\right]$,

where $\phi_{0}$ is the value of amplitude velocity at $z=z_{0}$. In this case, from Eq. (38) we find

$V_{a}^{2} \frac{d^{2} \Phi}{d z^{2}}-\left(k^{2} c_{\mathrm{s}}^{2}+k^{2} V_{a}^{2}-\omega^{2}\right) \Phi=0$,

or, using the solution (39), we get the dispersion equation

$\omega^{2}=k^{2}\left(c_{\mathrm{s}}^{2}+V_{a}^{2}\right)-\frac{V_{a}^{2}}{\mathfrak{x}^{2} H_{g}^{2}}$.

For the ionospheric F-layers, at the levels $\sim(250-300) \mathrm{km}$, the height of the homogeneous atmosphere $H=(50-60) \mathrm{km}$ and we may get $V_{a}=(3-5) \mathrm{km} / \mathrm{s}$. Then for the value $\mathfrak{x}=1.4$, we can evaluate that for both heights, the last term in Eq. (41) is negligibly small. Thus, we may conclude that in the ionospheric F-layer at the horizontal propagation, we may always neglect the contribution from AGW, i.e. the term $V_{a}^{2} \frac{d^{2} \Phi}{d z^{2}}$ (see the last term in Eq. 41).

The case $X \neq 0$ needs a special consideration, but if we restrict ourselves (as in the formulae 8 and 9) with the almost transversal, with respect to $H_{0 z}$, propagation of AGW, when $\left|K_{z}\right|^{2} \ll k^{2}$, where $K_{z}=M+i k_{z}$ is the complex vertical wave-number, then in the differential operator $l_{H}^{2}=$ $k^{2} V_{a}^{2}-V_{a}^{2} d^{2} / d z^{2}$ we may also neglect the last term. Then from Eqs. (34) and (35), we easily find that each of the functions $\Phi$ and $X$ must satisfy the equation

$$
\begin{gathered}
\left(k^{2} V_{a}^{2}-\omega^{2}\right)\left(\frac{d^{2} F}{d z^{2}}+\frac{\beta+g}{?_{s}^{2}} \frac{d F}{d z}+\frac{\omega^{2}}{c_{s}^{2}} F\right) \\
-k^{2}\left(\frac{g \beta}{c_{\mathrm{s}}^{2}}-\omega^{2}\right) F=0 .
\end{gathered}
$$

Equation (42) exactly coincides with the Monin-Obukhov (1958) equation, where instead of the value $\omega_{a}^{2}=k^{2} V_{a}^{2}$ the Coriolis parameter $l=2 \omega_{0 z}$ exists. Therefore, we may directly write the dispersion Eq. (5) for AGW, taking into account the action of the geomagnetic field $\boldsymbol{H}_{\mathbf{0}}$ on the waves under consideration. Now the frequencies of the internal acoustic and gravitational waves in the ionospheric F-region are expressed with the following formulae:

$$
\begin{aligned}
\omega_{\mathrm{ac}}^{2} & =æ g \mathrm{H}\left(k^{2}+k_{z}^{2}+\frac{1}{4 H^{2}}+\frac{1}{L_{H}^{2}}\right) \\
& =k^{2}\left(c_{\mathrm{s}}^{2}+\frac{H_{0 z}^{2}}{4 \pi \bar{\rho}}\right)+c_{\mathrm{s}}^{2}\left(k_{z}^{2}+\frac{1}{4 H^{2}}\right),
\end{aligned}
$$




$$
\begin{aligned}
& \omega_{g}^{2}=\left.\frac{g}{H}\left(1-\frac{1}{\mathfrak{x}}\right)+\frac{H_{0 z}^{2}}{4 \pi \bar{\rho}}\left(k_{z}^{2}+\frac{1}{4 H^{2}}\right)\right] \\
& k^{2}\left(c_{\mathrm{s}}^{2}+\frac{H_{0 z}^{2}}{4 \pi \bar{\rho}}\right)+c_{\mathrm{s}}^{2}\left(k_{z}^{2}+\frac{1}{4 H^{2}}\right)
\end{aligned}
$$

where $L_{H}=c_{\mathrm{s}} / \omega_{a}=c_{\mathrm{s}} / k V_{a}$ is the characteristic scale of the horizontal motions of the compressible medium in the magnetic field of the Earth (analogue of the Obukhov scale $L_{0}=c_{\mathrm{s}}(\ell), c_{\mathrm{s}}^{2}=æ g H, H$ is the height of the homogeneous atmosphere.

In the absence of the magnetic field $V_{a}=H_{0 z} / \sqrt{4 \pi \bar{\rho}} \rightarrow$ $0, L_{H}=\infty$ formulae (43) and (44) passes into the classical expressions (7) for AGW (Khantadze, 1973). From the formulae (43) and (44) follows that in the ionospheric F-region geomagnetic field $\boldsymbol{H}_{\mathbf{0}}$ increases the height of the homogeneous atmosphere $H$ by $0.5 L_{H}$ and creates an additional dispersion in the gravitational branch. Acoustic branch (Eq. 43) contains longitudinal magneto-acoustic wave $k^{2}\left(c_{\mathrm{s}}^{2}+V_{a}^{2}\right)$, and gravitational branch (Eq. 44) - transverse Alfvén waves $V_{a}^{2}\left(k_{z}^{2}+1 /\left(4 H^{2}\right)\right)$. From Eq. (5) follows that gravitational waves in the ionosphere are always more lowfrequency (from $13 \mathrm{~min}$ up to several hours) than acoustic (up to $13 \mathrm{~min}$ ). At indifferent stratification of medium $(\mathfrak{x}=1)$, which, under the ionospheric conditions, is well fulfilled in the F-region (Khantadze, 1973; Jacchia, 1977), as it is seen from Eq. (44), under the action of geomagnetic field gravitational branch remains. In the ordinary atmosphere (when $H_{0 z}=0$ and $æ \rightarrow 1$ ) gravitational frequencies $\omega_{g}$, as it was shown above, are completely filtered. Numerous experiments confirm the existence of the gravitational type wavy perturbations (Sorokin and Fedorovich, 1982; Sharadze, 1991) in the ionospheric F-region.

\section{Planetary electromagnetic waves in the ionospheric E- and F-regions}

In the ionosphere, besides the MHD perturbations, in any season of a year global background wavy perturbations of electromagnetic nature also regularly exist having different spatial and temporal scales. Large-scale wave structures play an important role in the processes of general energy balance and circulation of the atmosphere and ocean. The large amount of observational data (Cavalieri et al., 1974; Cavalieri, 1976; Manson et al., 1981; Sorokin, 1988; Sharadze et al., 1988, 1989; Bauer et al., 1995; Zhou et al., 1997; Alperovich and Fedorov, 2007) verify the permanent existence of ULF electromagnetic planetary scale perturbations in Eand F-regions of the ionosphere. Among them, the ionospheric ultra-low-frequency (ULF) perturbations of planetary scale $\left(10^{3}-10^{4} \mathrm{~km}\right)$, propagating at fixed latitude along the Earth's parallel, are of special interest to the experimental observations (Burmaka et al., 2006).
As was noted above, the existence of the new branch of large-scale ULF wavy perturbations having an electromagnetic nature in the ionospheric E- and F-regions, was first theoretically predicted in Khantadze $(1986,2001)$. In the same paper, first the classification of the electromagnetic planetary waves (fast and slow waves) is given.

For the planetary scale waves instead of Euler equation in Eq. (13), it is necessary to use Fridman equation for vorticity, which naturally contains latitudinal gradients and curvature of the geomagnetic lines of force. Together with the induction equation (the third equation of the system 1) they form a closed system for perturbed velocity $\boldsymbol{V}$ and induced magnetic field $\boldsymbol{h}$ :

$\operatorname{helm} \boldsymbol{\Omega}=\boldsymbol{\Gamma}$, helm $\frac{\boldsymbol{H}}{\alpha \rho}=-\delta \boldsymbol{\Gamma}$,

where $\boldsymbol{H}=\boldsymbol{H}_{\mathbf{0}}+\boldsymbol{h}, \boldsymbol{\Gamma}=\nabla \times \nabla \times \boldsymbol{H}_{0} / 4 \pi \rho, \boldsymbol{\Omega}=\nabla \times \boldsymbol{V}$.

In this approximation the existence of waves having an electromagnetic nature at ionospheric levels is the major consequence of the ionospheric MHD Eq. (45) (Khantadze, 1973).

In the paper Khantadze (1999), it is shown that Eq. (45) in the "standard" coordinate system has an exact solution $V_{y}(x, t), V_{z}(x, t), h_{y}(x, t), h_{z}(x, t), H_{y}=H_{0 y}(y, z), H_{z}=$ $H_{0 z}(y, z)$ in the form of zonal electromagnetic planetary waves $V, h \sim \exp \left(-i \omega t+i k_{x} x\right)$ propagating along a parallel around the Earth. At the same time, for own frequencies, the following dispersion equation is obtained:

$\frac{\omega}{\omega_{H}}+\frac{\omega_{p}^{\prime}}{\omega}=\delta$,

where $\omega_{H}$ and $\omega_{p}^{\prime}$ are defined by the formulae:

$$
\begin{aligned}
\omega_{H} & =\frac{\alpha k_{x}}{4 \pi} \sqrt{\beta_{1}^{2}+\beta_{2}^{2}}=\frac{c H_{e}}{4 \pi e N} \frac{\sqrt{1+3 \sin ^{2} \theta^{\prime}}}{R} k_{x} ; \\
c_{H} & =\frac{\omega_{H}}{k_{x}} ; \\
\omega_{p}^{\prime} & =-\frac{\beta_{H}}{k_{x}}=-\frac{N}{N_{n}} \frac{e H_{e}}{M c} \frac{\sqrt{1+3 \sin ^{2} \theta^{\prime}}}{k_{x} R} \quad ; \quad c_{p}^{\prime}=\frac{\omega_{p}^{\prime}}{k_{x}} .
\end{aligned}
$$

Here $\alpha=c^{2} / H_{0} \sigma_{H} \approx c / e N$ is the Hall's parameter, $\sigma_{H}=$ $e N c / H_{0}$ is Hall's conductivity in the ionospheric Eregion, $k_{x}=2 \pi / \lambda$ is the wavenumber, $\beta_{1}=\partial H_{0 z} / \partial y, \beta_{2}=$ $\partial H_{0 y} / \partial y, \partial / \partial y=-R^{-1} \partial / \partial \theta^{\prime}, H_{0 z}=-2 H_{e} \cos \theta^{\prime}, H_{0 y}=$ $-H_{e} \sin \theta^{\prime}, R$ is the Earth's radius, $\theta^{\prime}=\pi / 2-\chi^{\prime}$ is the magnetic co-latitude, $H_{e}=0.32 \mathrm{G}$ is the value of the geomagnetic field at the equator. Neglecting the curvature of the geomagnetic lines of force, magnetic gradients $\beta_{1}$ and $\beta_{2}$ can be determined from Maxwell's equations: $\partial H_{0 z} / \partial y-\partial H_{0 y} / \partial z=$ $0, \partial H_{0 y} / \partial y+\partial H_{0 z} / \partial z=0$; the $\mathrm{x}$-axis is directed along the parallel west-east, the y-axis from south to north, and $\mathrm{z}-$ vertically upwards.

Calculations show that parameters of fast magnetogradient waves $c_{H}=\omega_{H} / k_{x}$, which are generated by electromagnetic gyroscopic force $\boldsymbol{F}_{H}$, in the ionospheric E-region 
$(\delta=1)$ are within the limits: $\lambda \sim\left(10^{3} \div 10^{4}\right) \mathrm{km}, \omega_{H} \sim$ $\left(10^{-1} \div 10^{-4}\right) \mathrm{s}^{-1}$, and $c_{H} \sim(1 \div 7) \mathrm{km} \mathrm{s}^{-1}$.

The propagation of fast electromagnetic planetary $c_{H^{-}}$ waves are accompanied by the significant pulsations of the geomagnetic field (20-80 nT). These oscillations in the middle and moderate latitudes were registered during the launching of spacecrafts (Burmaka et al., 2006) and by the world network of ionospheric and magnetic observatories (Alperovich and Fedorov, 2007; Sharadze et al., 1988; Sharadze, 1991). As it is seen from Eq. (47), such oscillations can exist also at higher and lower latitudes $\chi^{\prime}$ i.e. they have a general-planetary nature. In these waves, like in helicons, only electrons are oscillating while the ions and neutrals are immovable. From Eq. (48), it follows that in the neutral and ion components due to the total drag, $V_{i}=V_{a}$, in the ionospheric E-region slow planetary ULF Rossby-type waves are also excited (Tolstoy, 1967; Khantadze, 1967). Parameters of slow waves are within the limits $\lambda \sim\left(10^{3}-10^{4}\right) \mathrm{km}$, $\omega_{p}^{\prime} \sim\left(10^{-4}-10^{-5}\right) \mathrm{s}^{-1}, c_{p}^{\prime}=\omega_{p}^{\prime} / k_{x} \sim(100-300) \mathrm{m} \mathrm{s}^{-1}$. The amplitude variation of the geomagnetic field reaches 1-20 nT. In these waves, ions and neutrals are oscillating while electrons are immovable. These slow, weather-forming waves were discovered by ionospheric observations (Cavalieri et al., 1974; Sharadze and Khantadze, 1979; Sharadze et al., 1989; Sharadze, 1991).

In the ionospheric F-region $(\delta=0)$, taking into account the identical equality $\omega_{H} \omega_{p}^{\prime}=-\omega_{n}^{2}$, for the new mode of the eigen-frequency we obtain from Eq. (46):

$\omega_{n}=\frac{H_{e}}{\sqrt{4 \pi \rho}} \frac{\sqrt{1+3 \sin ^{2} \theta^{\prime}}}{R}$.

In these standing waves, under the action of the force $\boldsymbol{F}_{H}$, the ionospheric medium is oscillating as a whole. Characteristic values of the wave parameters are changing in the following range $\lambda \sim\left(10^{3}-10^{4}\right) \mathrm{km}, \omega_{n} \sim 3 \times 10^{-3} \mathrm{~s}^{-1}, c_{n}=$ $\omega_{n} / k_{x} \sim(5-45) \mathrm{km} \mathrm{s}^{-1}$. The amplitude of the geomagnetic pulsations in these electromagnetic planetary waves varies from 10 to several tens of nT. Experimentally these waves are detected at the middle latitudes in the ionospheric F-region (Sharadze et al., 1988; Sorokin, 1988; Sharadze, 1991; Bauer et al., 1995; Fagundes et al., 2005). Maximum parameter values of considered waves are observed at the magnetic equator.

Note that when we neglect the curvature of the lines of force (formulae 47-49), the geomagnetic field will differ from dipole field to within $20 \%$. Observations show (Eleman, 1973) that the deviation of the geomagnetic field from the dipole manifests itself only at a distance of several tens of thousands of kilometres. Therefore, the above-mentioned expressions for planetary waves are only approximate formulae. An attempt to fill this gap was made in the papers (Aburjania et al., 2003, 2004, 2005; Aburjania and Khantadze, 2005; Khantadze et al., 2006).

With the taking into account the curvature of the geomagnetic lines of force in the spherical coordinate system was shown (Khantadze et al., 2006) that there exists an exact solution of fundamental system (45) in the form of magnetogradient zonal planetary waves, $V\left(\lambda^{\prime}, t\right), h\left(\lambda^{\prime}, t\right), H_{0 r}\left(r, \theta^{\prime}\right)$, $H_{0 \theta}\left(r, \theta^{\prime}\right), H_{0 \lambda^{\prime}}=0$ :

$c_{H}=0,5 \frac{c H_{e}}{4 \pi e N} \frac{\sin \theta^{\prime} \pm \sqrt{24+\sin ^{2} \theta^{\prime}}}{R}$,

$c_{p}^{\prime}=-0,5 \frac{N}{N_{n}} \frac{e H_{e}}{M c} \frac{-\sin \theta^{\prime} \pm \sqrt{24+\sin ^{2} \theta^{\prime}}}{k^{2} R}$,

$c_{n}=0,5 \frac{H_{e}}{\sqrt{4 \pi \rho}} \frac{-\sin \theta^{\prime} \pm \sqrt{24+\sin ^{2} \theta^{\prime}}}{k R}$,

where $k=2 \pi / \lambda, \lambda$ is the wavelength of the planetary wave. The relation $m=k R \sin \theta^{\prime}=(2 \pi R / \lambda) \sin \theta^{\prime}$ shows how many waves fit on the magnetic latitude $\chi^{\prime}$ (we assume that the magnetic moment is combined with the Earth's rotation axis). At $m=1$ one wavelength fits around the parallel, at $m=2-$ two, etc. Observations show that in the ionospheric E- and F-regions planetary waves with the zonal wave-numbers $m=2 \div 10$ (Sorokin and Federovich, 1982; Sharadze, 1991) regularly exist.

From the formulae (50-52) follows the important conclusion: geomagnetic field stratifies ionospheric plasma along the direction $\chi^{\prime}$ as the gravity force stratifies the atmosphere along altitude. The waves move along the parallel with the different phase velocities in both the east and west directions. For example, as it follows from the formula (52), at the equator $\left(\theta^{\prime}=\pi / 2\right)$, where the phase velocity of the wave reaches maximum value, for the waves, propagating in a west-east direction $\left(c_{n}>0\right)$, we obtain: $c_{n+}=2 c_{0}$, where $c_{0}=\left(H_{e} / \sqrt{4 \pi \rho}\right) / k R$. For the waves moving in an eastwest direction $\left(c_{n}<0\right)$, we obtain: $c_{n-}=-3 c_{0}$. It should be noted that this important property of magnetogradient waves was predicted by Kobaladze and Khantadze (1989). The physical mechanism of generating these new free oscillations, for example in the F-region, follows from the simplified equations: $i \omega V_{\theta^{\prime}}=\left(\beta_{1} / 4 \pi \rho\right) h_{r}, i \omega h_{r}=\beta_{1} V_{\theta^{\prime}}$, where $\beta_{1}=-\left(\partial H_{0 r} / \partial \theta^{\prime}\right) / R=2 H_{e} \sin \theta^{\prime} / R$. Indeed, introducing the transverse displacement of medium particles $\xi_{\theta^{\prime}}, V_{\theta^{\prime}}=d \xi_{\theta^{\prime}} / d t$, and the specific quasi-elastic electromagnetic force $f=\left(\beta_{1} / 4 \pi \rho\right) h_{r}=-\left(\beta_{1}^{2} / 4 \pi \rho\right) \xi_{\theta^{\prime}}$ from this simplified system, we easily obtain the equation of the free oscillations for the linear oscillator $d^{2} \xi_{\theta^{\prime}} / d t^{2}+\omega_{0}^{2} \xi_{\theta^{\prime}}=0$, where $\omega_{0}$ is the eigen frequency of the oscillator, $\omega_{0}^{2}=\omega_{n}^{2}=$ $\left(\beta_{1}^{2} / 4 \pi \rho\right)=k / \rho$. At the same time from the freezing-in condition $h_{r}=-\beta_{1} \xi_{\theta^{\prime}} \approx\left(H_{0 r} / R\right) \xi_{\theta^{\prime}}$ follows that all transverse displacement of neutral particle $\xi_{\theta^{\prime}}$ in the F-region due to the collisions with plasma particles generates tension in the ionospheric plasma of lines of force of the geomagnetic field $H_{0 r}$. As a result in the magnetic field $H_{0 r}$, proportional to $\xi_{\theta^{\prime}}$ perturbation $h_{r}$ will arise being a cause of excitation of electromagnetic quasi-elastic force $f=\left(-\beta_{1}^{2} / 4 \pi \rho\right) \xi_{\theta^{\prime}}=-(k / \rho) \xi_{\theta^{\prime}}$. Here the value $k=\beta_{1}^{2} / 4 \pi$ 
can be called the coefficient of electromagnetic elasticity of the ionospheric medium. Waves (50-52) are discussed in more details in Kobaladze and Khantadze (1989); Petviashvili and Pokhotelov (1992); Khantadze et al. (2006).

Note that the peculiarities of propagation of internal gravity waves and so-called magnetized Rossby waves (MRW) in the ionospheric E-layer was investigated recently (Kaladze, 1999; Kaladze et al., 2004; Kaladze and Horton, 2006; Horton et al., 2008). The term MRW was introduced by Kaladze (1999) for the ionospheric generalization of tropospheric Rossby waves in a rotating atmosphere by the spatially inhomogeneous geomagnetic field. The MRW belong to the ULF range $\left(10^{-5}-10^{-4}\right) \mathrm{s}^{-1}$, with the wavelength of the order $1000 \mathrm{~km}$ and longer, and the phase velocity is the velocity of the local winds, i.e. $\sim(1-100) \mathrm{ms}^{-1}$. MRW do not significantly perturb the geomagnetic field. For the typical ionization fraction in the E-layer, the Ampere force is comparable to the Coriolis force, both having a spatial inhomogeneity scale of the Earth's radius. Thus, they are induced by the latitudinal inhomogeneity both of the Earth's angular velocity and of the geomagnetic field. The Ampere force opposes the Coriolis force vorticity and, therefore, partial or full compensation of the Coriolis deviation by the magnetic deviation is possible. Correspondingly, the propagation phase velocity of the linear waves also decreases. The MRW are excited solely by the ionospheric dynamo electric field when the Hall effect, due to the interaction with the ionized ionospheric component in the E-layer, is induced.

The influence of Raleigh friction on the damping rate of the planetary electromagnetic waves in the ionospheric Elayer was considered in papers by Kaladze et al. (2003) and Kaladze (2004). The nature of the three-dimensional planetary electromagnetic waves propagating in the Earth's ionosphere was investigated by Khantadze et al. $(2004,2009)$ and Khantadze and Jandieri (2009). Spherical geometry of the planetary electromagnetic waves in the Earth's ionosphere was investigated by Khantadze et al. (2008).

\section{Conclusions}

To summarize, it can be concluded that, in the MHD approximation (with dissipation and temperature stratification taken into account), the ionosphere as a cavity is described by a set of differential equations of the eighth order in time. The system of Eq. (1) gives six scalar equations for the velocity $\boldsymbol{V}$, and the magnetic field $\boldsymbol{H}$. The density $\rho$ and pressure $P$ are described by two scalar equations: the continuity equation and the energy equation for polytropic processes. Consequently, a slightly perturbed ionosphere has eight eigen-frequencies: the first two frequencies $\omega_{1,2}$ represent the acoustic branch, containing an ordinary acoustic wave and due to the elasticity of the geomagnetic field lines - magneto-acoustic wave (with its limiting case - helicons (atmospheric whistlers); the second pair of frequen- cies are the internal gravitational waves $\omega_{3,4}$; the fifth frequency $\omega_{5}$ belongs to the planetary Rossby waves; the third pair of frequencies $\omega_{6,7}$ represents the slow MHD Alfvénlike waves due to the tension of the geomagnetic field lines (a limiting case of these waves are slow ion cyclotron waves with the frequency $\eta \omega_{i}$ ); and the eighth eigen-frequency $\omega_{8}$, was discovered by Khantadze $(1986,1999,2001)$. In the ionospheric E-region, this eighth eigen-frequency is equal to $\omega_{8}=\omega_{H}$ for fast planetary waves with the velocity $c_{H}$ in the electronic plasma component and to $\omega_{8}=\omega_{p}^{\prime}$ for slow planetary Rossby-like waves in the ion plasma component. In the ionospheric F-region, this eigen-frequency is equal to $\omega_{8}=\omega_{n}-$ a frequency at which the ionospheric medium with a density of $\rho_{e}+\rho_{i}+\rho_{n} \approx \rho_{n}$ oscillates as a single entity and propagates with the velocity $c_{n}$ in the form of fast planetary waves. At mid-latitudes, these waves in the ionospheric Eregion show up as well-observed, large-scale perturbations, or mid-latitude long-period oscillations (MLOs), and in the F-ionospheric region, they show up as magneto-ionospheric wave perturbations (MIWPs). A broad variety of properties of the magneto-gradient planetary waves considered above holds great promise for a more detailed investigation of the large-scale electromagnetic perturbations regularly observed both in a quiet (background oscillations) and a perturbed (induced oscillations) ionosphere in the E- and F-regions.

Thus, all eigen-frequencies of the ionosphere are fully covered when the oscillating system of the three-component ionospheric plasma is described by the system of MHD partial differential equations of the eighth order with respect to time.

We would like to emphasize again that unlike the pure plasma, where $\eta=N /\left(N_{n}+N\right)=1$, in the weakly-ionized ionosphere magneto-acoustic and Alfvén waves are slow MHD waves (Khantadze, 1971; Khantadze and Sharadze, 1980; Sorokin and Federovich, 1982).

This article is dedicated to the memory of Professor A.G. Khantadze who passed away in November 2009.

Acknowledgements. The research described in this publication was made possible in part by Award No. GNSF/ST08/5-442 of Georgian National Science Foundation and ISTC grant \# G-1376.

Topical Editor C. Jacobi thanks two anonymous referees for their help in evaluating this paper.

\section{References}

Aburjania, G. D., Jandieri, G. V., and Khantadze, A. G.: Self - organization of planetary electromagnetic waves in E-region of the ionosphere, J. Atmos. Solar-Terr. Phys., 65(6), 661-671, 2003.

Aburjania, G. D., Chargazia, K. Z., Jandieri, G. V., Khantadze, A. G., and Kharshiladze, O. A.: On the new modes of planetaryscale electromagnetic waves in the ionosphere, Ann. Geophys., 22, 1203-1211, doi:10.5194/angeo-22-1203-2004, 2004.

Aburjania, G. D., Chargazia, Kh. Z., Jandieri, G. V., Khantadze, A. G., Kharshiladze, O. A., and Lominadze, J. G.: Generation and propagation of the ULF planetary-scale electromagnetic wavy 
structures in the ionosphere, Planet. Space Sci., 53(9), 881-901, 2005.

Aburjania, G. D. and Khantadze, A. G.: Specific features of the propagation of ULF planetary electromagnetic waves in the earth's ionosphere related to the geomagnetic field curvature, Geomagnetism and Aeronomy, 45(5), 633-640, 2005.

Alperovich, L. S. and Fedorov, E. N.: Hydromagnetic Waves in the Magnetosphere and the Ionosphere, Springer, 426 p., 2007.

Bauer, T. M., Baumjohann, W., Treumann, R. A., et al.: Lowfrequency waves in the near-Earth plasma sheet, J. Geophys. Res., 100A, 9605-9617, 1995.

Becker, E. and Schmitz, G.: Energy deposition and turbulent dissipation owing to gravity waves in the mesosphere, J. Atmos. Sci., 59, 54-68, 2002.

Burmaka, V. P., Lysenko, V. N., Chernogor, L. F., and Chernyak, Yu. V.: Wave-like processes in the ionospheric F region that accompanied rocket launches from the Baikonur Site, Geomagnetism and Aeronomy, 46(6), 742-759, 2006.

Cavalieri, D. J., Deland, R. J., Poterna, J. A., and Gavin, R. F.: The correlation of VLF propagation variations with atmospheric planetary-scale waves, J. Atmos. Terr. Phys., 36, 561-574, 1974.

Cavalieri, D. J.: Traveling planetary-scale waves in the E-region, J. Atmos. Terr. Phys., 38, 965-978, 1976.

Eleman, P.: Geomagnetic Field, Cosmical Geophysics, edited by: Egelend, A., Houlter, O., and Omholt, A., Oslo, Bergen, Tromso, Universitets forlaget, 1973.

Fagundes, P. R., Pillat, V. G., Bolzan, M. A., et al.: Observations of $\mathrm{F}$ layer electron density profiles modulated by planetary wave type oscillations in the equatorial ionospheric anomaly Region, J. Geophys. Res., 110, 1302-1312, 2005.

Fridman, A. A.: Experiment in the Hydromechanics of a Compressible Fluid, Gostekhizdat, Moscow, 1934 (in Russian).

Fritts, D. C. and Alexander, M. J.: Gravity wave dynamics and effects in the middle atmosphere, Rev. Geophys., 41(1), 10031066, doi:10.1029/2001RG000106, 2003.

Garcia, R. R. and Solomon, S.: The effect of breaking waves on the dynamics and chemical composition of the mesosphere and lower thermosphere, J. Geophys. Res., 90, 3850-3868, 1985.

Grigor'ev, G. I.: Acoustic-gravity waves in the earth's atmosphere (review), Radiophysics and Quantum Electronics, 42(1), 1-21, 1999.

Hajkowicz, L. A.: Global onset and propagation of large-scale traveling ionospheric disturbances as a result of the great storm of 13 March 1989, Planet. Space Sci., 39, 583-593, 1991.

Hines, C. O.: Internal atmospheric gravity waves at ionospheric heights, Can. J. Phys., 38, 1441-1481, 1960.

Holton, J. R.: The role of gravity wave induced drag and diffusion in the momentum budget of the mesosphere, J. Atmos. Sci., 39, 791-799, 1982.

Holton, J. R.: The influence of gravity wave breaking on the general circulation of the middle atmosphere, J. Atmos. Sci., 40, 24972507, 1983.

Hooke, W. H.: Ionospheric irregularities produced by internal atmospheric gravity waves, J. Atmos. Solar-Terr. Phys., 30, 795-823, 1968.

Horton, W. C., Kaladze, T. D., Van Dam, J. W., and Garner, T. W.: Zonal flow generation by internal gravity waves in the atmosphere, J. Geophys. Res., 113, A08312, doi:10.1029/2007JA012952, 2008.
Houghton, J. T.: The stratosphere and mesosphere, Quarterly Journal of the Royal Meteorological Society, 104, 1-29, 1978.

Jacchia, L. G.: Thermospheric temperature, density and composition: new models, Spec. Rep. Smithsonian Astrophys. Observ. 375, 1-106, 1977.

Kadomtsev, B. B.: Collective phenomena in plasmas, Pergamon Press, New York, 1982.

Kaladze, T. D.: Magnetized Rossby waves in the Earth's ionosphere , Plasma Physics Reports, 25(4), 284-287, 1999.

Kaladze, T. D., Pokhotelov, O. A., Sagdeev, R. Z., Stenflo, L., and Shukla, P. K.: Planetary electromagnetic waves in the ionospheric E-layer, J. Atmos. Solar-Terr. Phys., 65, 757-764, 2003.

Kaladze, T. D.: Planetary electromagnetic waves in the ionospheric E-layer, Proceedings of the First Cairo Conference on Plasma Physics \& Applications: CCPPA 2003 (Cairo, Egypt, October 11-15, 2003). Shriften des Forschungszentrums Jülich, Bilateral Seminars of the International Bureau, edited by: Kunze, H.-J., El-Khalafawy, T., and Hegazy, H., German-Egyptian Cooperation, 34, 68-74, 2004.

Kaladze, T. D., Aburjania, G. D., Kharshiladze, O. A., Horton, W., and Kim, Y.-H.: Theory of magnetized Rossby waves in the ionospheric E layer, J. Geophys. Res., 109, A05302, doi:10.1029/2003JA010049, 2004.

Kaladze, T. D. and Horton, W.: Synoptic-scale nonlinear stationary magnetized Rossby waves in the ionospheric E-layer, Plasma Physics Reports, 32(12), 996-1006, 2006.

Kaladze, T. D., Pokhotelov, O. A., Stenflo, L., Shah, H. A., and Jandieri, G. V.: Electromagnetic inertio-gravity waves in the ionospheric E-layer, Physica Scripta, 76, 343-348, 2007.

Kaladze, T. D., Pokhotelov, O. A., Shah, H. A., Khan, M. I., and Stenflo, L.: Acoustic-gravity waves in the earth's ionosphere, J. Atmos. Solar-Terr. Phys., 70(13), 1607-1616, 2008.

Kamide, Y. and Baumjohann, W.: Magnetosphere-Ionosphere Coupling, Berlin: Springer, 1993.

Kelley, M. C.: The Earth's Ionosphere, Academic, San Diego, Calif., 1989.

Khantadze, A. G.: Determination of the wind field by the pressure gradient field and latitudinal effect of geomagnetic field, Proc. Inst. Geophys. Acad. Sci. Georgian SSR, 24-29, 1967 (in Russian).

Khantadze, A. G.: About internal waves in conducting atmosphere, Bull. Acad. Sci. Georgian SSR, 61(3), 133-136, 1971 (in Russian).

Khantadze, A. G.: On the dynamics of conducting atmosphere, Nauka, Tbilisi, 267 p., 1973 (in Russian).

Khantadze, A. G. and Sharadze, Z. S.: Ionospheric effects of planetary waves, Alma-Ata: Nauka, 143 p., 1980.

Khantadze, A. G., Kobaladze, Z. A., and Pataraya, A. D.: Excitation of soliton Rossby waves by internal gravity waves, Doklady Earth Sci., 262(5), 1083-1091, 1982.

Khantadze, A. G.: Hydromagnetic gradient waves in dynamo region of the ionosphere, Bull. Acad. Sci. Georgian SSR, 123(1), 69-71, 1986 (in Russian).

Khantadze, A. G.: On the electromagnetic planetary waves in the Earth's ionosphere, J. Georgian Geophys. Soc., 4B, 125-127, 1999 (in Russian).

Khantadze, A. G.: On a new branch of natural oscillations in a conductive atmosphere, Doklady Earth Sci., 376(2), 250-252, 2001.

Khantadze, A. G., Aburjania, G. D., and Jandieri, G. V.: Mechanism 
for the Generation of a Vortex Electric Field in the ionospheric E-Region, Plasma Physics Report, 30(1) 83-90, 2004.

Khantadze, A. G., Aburjania, G. D., and Lominadze, J. G.: New branches of natural ULF electromagnetic oscillations of the ionospheric resonator, Doklady Earth Sci., 406(2) 244-258, 2006.

Khantadze, A. G., Jandieri, V. G., and Jandieri, G. V.: Planetary waves in rotating ionosphere, Plasma Physics Reports, 34(6), 480-485, 2008.

Khantadze, A. G., Lominadze, J. G., and Jandieri, G. V.: Internal waves in the ionosphere caused by the Earth's geomagnetic field, Doklady Earth Sci., 420(4), 672-675 (2008).

Khantadze, A. G. and Jandieri, G. V.: General-planetary character of three-dimensional planetary waves propagation in lower and upper earth atmosphere, J. Atmos. Solar-Terr. Phys., 71, 45-48, 2009.

Khantadze, A. G., Jandieri, G. V., Ishimaru, A., and Jandieri, V. G.: Planetary waves and vortex structures in the ionosphere, Ann. Geophys., 27, 3489-3495, doi:10.5194/angeo-27-34892009, 2009.

Kobaladze, Z. L. and Khantadze, A. G.: About propagation of largescale perturbations in the ionosphere, Bull. Acad. Sci. Georgian SSR, 134(1), 97-100, 1989 (in Russian).

Kulsrud, R. M.: Plasma Physics for Astrophysics, Princeton University Press, 2005.

Lindzen, R. S.: Turbulence and stress owing to gravity wave and tidal breakdown, J. Geophys. Res., 86, 9707-9714, 1981.

Lübken, F.-J.: Seasonal variation of turbulent energy dissipation rates at high latitudes as determined by in situ measurements of neutral density fluctuations, J. Geophys. Res., 102, 1344113456, 1997.

Lysak, R. L., Song, Y., and Jones, T. W.: Propagation of Alfvén waves in the magnetotail during substorms, Ann. Geophys., 27, 2237-2246, doi:10.5194/angeo-27-2237-2009, 2009.

Manson, A. H., Heek, C. E., and Gregory, J. B.: Winds and waves (10 min-30 day) in the mesosphere and lower thermosphere at Saskatoon, J. Geophys. Res., 86, 9615-9625, 1981.

McLellan, F. and Winterberg, A.: Magneto-acoustic and magnetogravitation waves, Solar Phys., 4, 401-430, 1968.

Monin, A. S. and Obukhov, A. M.: Small oscillations of the atmosphere and adaptation of meteorological fields, Izv. Acad. Nauk Geol., 11, 1360-1373, 1958 (in Russian).

Müllemann, A., Rapp, M., and Lübken, F.-J.: Morphology of turbulence in the polar summer mesopause region during the MIDAS/SOLSTICE campaign 2001, Adv. Space Res., 31, 20692074, 2003.
Prandtl, L.: Essentials of fluid mechanics. Haffner, New York, 1952.

Petviashvili, V. I. and Pokhotelov, O. A.: Solitary Waves in Plasmas and in the Atmosphere. Gordon and Beach Science publishers, 1992.

Pokhotelov, O. A., Parrot, M., Fedorov, E. N., Pilipenko, V. A., Surkov, V. V., and Gladychev, V. A.: Response of the ionosphere to natural and man-made acoustic sources, Ann. Geophys., 13, 1197-1210, doi:10.1007/s00585-995-1197-2, 1995.

Pokhotelov, O. A., Kaladze, T. D., Shukla, P. K., and Stenflo, L.: Three dimensional solitary vortex structures in the upper atmosphere, Physica Scripta, 64, 245-252, 2001.

Shaefer, L. D., Rock, D. R., Lewis, J. P., et al.: Detection of explosive events by monitoring acoustically-induced geomagnetic perturbations, Lawrence Livermore Laboratory, CA USA, 94550, 1999.

Sharadze, Z. S. and Khantadze, A. G.: Planetary waves in the ionospheric E and F regions, Bull. Acad. Sci. Georgian SSR, 94(1), 69-73, 1979 (in Russian).

Sharadze, Z. S., Japaridze, G. A., Kikvilashvili, G. B., et al.: Wavy disturbances of non-acoustical nature in the middle-latitude ionosphere, Geomag. Aeron., 28(3), 446-451, 1988.

Sharadze, Z. S., Mosiashvili, N. V., Pushkova, G. N., and Yudovich, L. A.: Long-period-wave disturbances in $\mathrm{E}$ region of the ionosphere, Geomag. Aeron., 29(3), 1032-1034, 1989.

Sharadze, Z. S.: Phenomena in the middle-latitude ionosphere, $\mathrm{PhD}$ Thesis, Moscow, 1991.

Sorokin, V. M. and Fedorovich, G. V.: Physics of slow MHD waves in the ionospheric plasma, Energoizdat, Moscow, 1982 (in Russian).

Sorokin, V. M.: Wavy processes in the ionosphere associated with geomagnetic field, Izv. Vuz. Radiofis., 31, 1169-1179, 1988 (in Russian)

Stenflo, L. and Shukla, P. K.: Nonlinear acoustic-gravity waves, J. Plasma Phys., 75(06), 841-847, 2009.

Tolstoy, I.: Hydromagnetic gradient waves in the ionosphere, J. Geophys. Res., 72(5), 1435-1442, 1967.

Yaglom, A. M.: Dynamics of large-scale processes in the barotropic atmosphere, Izv. Acad. Nauk. Geol., 4, 346-369, 1953 (in Russian).

Zhou, Q. H., Sulzer, M. P., and Tepley, C. A.: An analysis of tidal and planetary waves in the neutral winds and temperature observed at low-latitude E-region heights, J. Geophys. Res. 102(A6), 11491-11505, 1997. 